\title{
The Scientific Potential of LOFAR for Time Domain Astronomy
}

\author{
Rob Fender ${ }^{1}$, on behalf of the LOFAR Transients Key Science Project \\ ${ }^{1}$ School of Physics \& Astronomy, University of Southampton, SO17 1BJ, UK For TKSP \\ member list see: www.transientskp.org/team.shtml \\ email: r.fender@soton.ac.uk
}

Invited Talk

\begin{abstract}
LOFAR is a ground-breaking low-frequency radio telescope that is currently nearing completion across northern Europe. As a software telescope with no moving parts, enormous fields of view and multi-beaming, it has fantastic potential for the exploration of the timevariable universe. In this brief paper I outline LOFAR's capabilities, our plans to use it for a range of transient searches, and some crude estimated rates of transient detections.
\end{abstract}

Keywords. accretion, stars:binaries, stars:pulsars, stars:supernovae:general, ISM: jets and outflows, radio continuum: general

\section{Introduction}

LOFAR, the Low Frequency Array, is a large low-frequency radio telescope in northern Europe, led by ASTRON. Construction of the array, which has its core collecting area in The Netherlands with international stations in France, Germany, Sweden and the UK, is nearly complete, and astronomically interesting data are now being taken. LOFAR operates in the 30-80 and 120-240 MHz frequency ranges. The $80-120 \mathrm{MHz}$ frequency gap corresponds to the FM radio bands - frequencies at which astronomical observations are impossible†. Construction of the array is almost complete; Fig. 1 indicates the distribution of operating LOFAR stations across Europe. In addition, observations are occasionally possible to frequencies as low as $15 \mathrm{MHz}$.

LOFAR has six key science projects (KSPs), one of which is Transients (principal investigators Fender, Stappers \& Wijers). The remit of the TKSP covers all transient and variable astrophysics, including commensal searches of all data (ultimately in near-realtime, although that functionality is not yet implemented). The TKSP covers both timeseries and image-plane searches for transients and variables, including pulsars (Stappers et al. 2011). The adoption of transients and variables as key science drivers for the project is a theme for most of the large SKA pathfinders and precursors, and in that respect is generally unlike older radio facilities. However, time-series and image-plane transients have been separated for both ASKAP (which has CRAFT and VAST respectively) and MeerKAT (TRAPUM and ThunderKAT). That makes some sense from the aspect of techniques, although there is some overlap in the science.

$\dagger$ Unless northern Europe could be persuaded to stop night-time FM radio broadcasts for a few weeks in the interests of finding the Epoch of Reionisation signal.. 


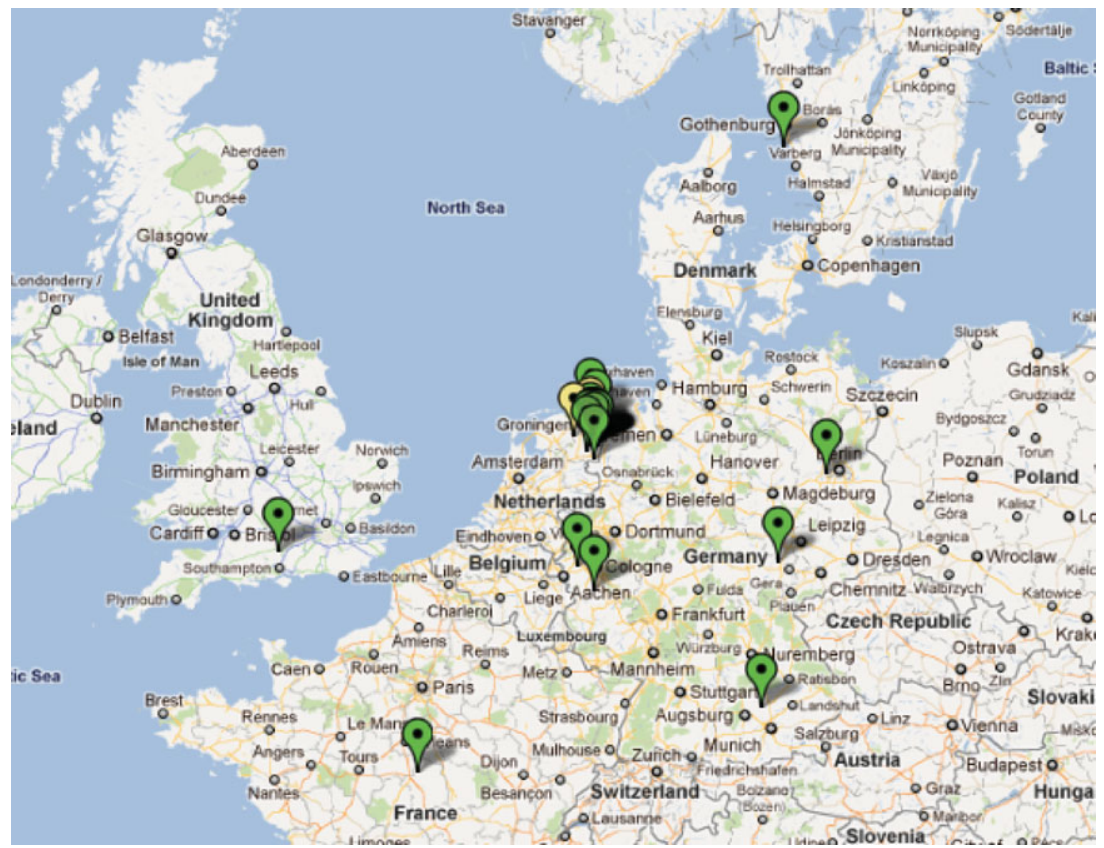

Figure 1. The distribution of complete LOFAR stations across Europe.

\section{Types of Radio Transients}

What are these transients that we're looking for? In planning transients searches, whether via targeted surveys or blind commensal studies, we can crudely separate events into astrophysics and technology.

Incoherent synchrotron emission arises from the acceleration of relativistic electrons in a magnetic field. It has a brightness temperature (intensity) limit of $\sim 10^{12} \mathrm{~K}$, and is associated with practically every event which injects kinetic energy into the ambient medium (e.g. jets of all types, nova and supernova explosions). Those events tend to evolve relatively slowly, especially at low frequencies (where the optical depth is higher), and so with LOFAR we do not expect to see variability on time-scales shorter than the standard imaging timescale of 1-10 sec.

Coherent emitters, such as radio pulsars or masers, can have much higher brightness temperatures. Like synchrotron events they can at times be associated with extreme astrophysical environments. The much higher brightness temperatures means that the variability time-scales can be much shorter, and even astrophysically distant objects can vary on time-scales much shorter than the standard imaging one. However, such short bursts are dispersed and scattered by the intervening interstellar medium, and need to be corrected for in studying the intrinsic properties of the burst (a well-understood problem in the field of radio pulsars). Note that there can be considerable overlap between these two sets of objects and techniques: for instance, variability in coherent sources can be detected and tracked in images.

Fig. 2 summarises this dichotomy in radio transients, and is indicative of how early attempts at automated classification pipelines might make an early branch based upon variability time-scales and polarisation characteristics. 


\section{Incoherent synchrotron emission}

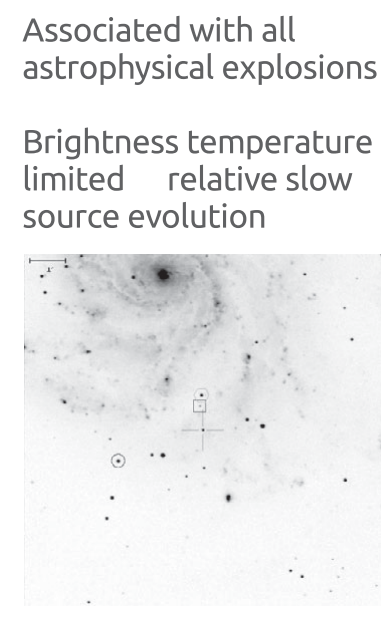

Mainly found in images

\section{Coherent emission}

\author{
Can have very high brightness \\ temperatures very rapid, \\ dispersed, variability, and be \\ highly polarized
}

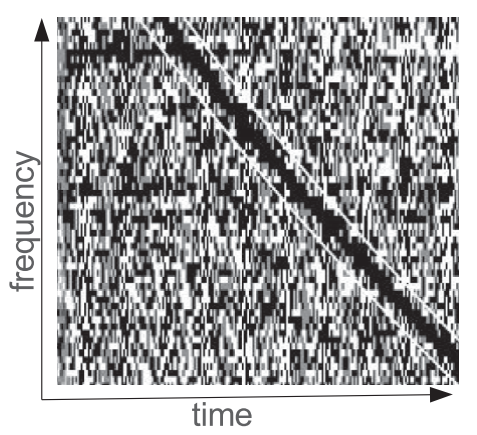

Mainly found in time series (but also in rapid imaging)

Figure 2. Transients can be divided into incoherent synchrotron and coherent events, which also corresponds roughly to a divide in the techniques used to find them (image plane vs. time series).

\section{Finding Transients with LOFAR}

LOFAR can operate in a variety of modes, all of which can be important for the study of transients and variables. Furthermore, all of those modes can be operated at a variety of levels, from a single station to the entire pan-European array.

Interferometric mode. LOFAR is a 'software telescope' which in effect has no moving parts. Pointing of the array and/or individual stations is done by introducing delays appropriate to a certain direction on the sky (phased array). Different frequencies can be therefore set to observe in different directions by introducing different delays. LOFAR can already, as a standard imaging mode, produce 8 beams each of $6 \mathrm{MHz}$ bandwidth. In the low band those beams can be placed anywhere on the sky; in the high band they are limited by the beam of the high-band tiles, an analogue beamformer. That allows for an extraordinary instantaneous field of view: $8 \times 90=720 \mathrm{deg}^{2}$ in the low band and $8 \times 25=200 \mathrm{deg}^{2}$ in the high band. In other words, the entire northern hemisphere could be mapped in the low band in less than 30 sets of pointings (with sparse tiling). Initial processing of wide-field surveys for transients, including the MSSS (Multifrequency Snapshot Sky Survey) due between late 2011 and early 2012, will only localise sources to a few arcmin, but later and/or responsive observations could localise interesting sources (including transients) to arcsecond precision.

Timing mode. LOFAR also has high-time-resolution ('pulsar') modes, which can achieve 10s of ns time resolution and can map either a full field of view with sensitivity $s \propto N^{-1 / 2}$ (incoherent sum), or the synthesised beam with sensitivity $s \propto N^{-1}$ (where smaller $s$ is better). Recently it has been possible to record data from over 100 coherent 
tied-array beams simultaneously and to tile out the entire HBA field. Stappers et al. (2011) give more details about searches for fast transients with LOFAR.

Direct storage. The LOFAR Transients Buffer Boards (TBBs) can be used to record up to several seconds of full bandwidth antenna level data (or longer, in a trade-off with bandwidth), before the beam-forming stage. That means that beams can be formed retrospectively in a certain direction anywhere in the sky (LBA) or tile beam (HBA) upon receipt of an 'internal' alert (from LOFAR itself) or an 'external' one (e.g. from VOEvent). That mode is currently being developed by the Cosmic Rays KSP (PI Falcke).

\section{LOFAR in a Global Context}

As noted above, LOFAR, ASKAP and MeerKAT have all embraced the science of radio transients as part of their Key Science Programmes. To that list we hope to add APERTIF, the focal-plane array upgrade to WSRT, to which several transients-oriented proposals have already been submitted as statements of interest for its KSP programme.

In a global context, LOFAR has the widest field of view of any of the major facilities, and although its suffers in terms of raw mJy sensitivity compared to (say) EVLA and MeerKAT, when a spectral correction is made it can be shown to be a very powerful facility. That is illustrated by Fig 3, where sensitivity and field of view are compared for a range of world-class radio facilities. The solid diagonal lines correspond to constant figures of merit (FoM, defined as FoM $\propto \Omega s^{-2}$, where $\Omega$ is the field of view and $s$ is the sensitivity; smaller $s$ implies increased sensitivity). The four new or upgraded GHz facilities - EVLA, MeerKAT, APERTIF and ASKAP - all have a comparable FoM. For LOFAR it is not until a spectral correction is made that its survey power becomes apparent, as the Figure caption explains in more detail.

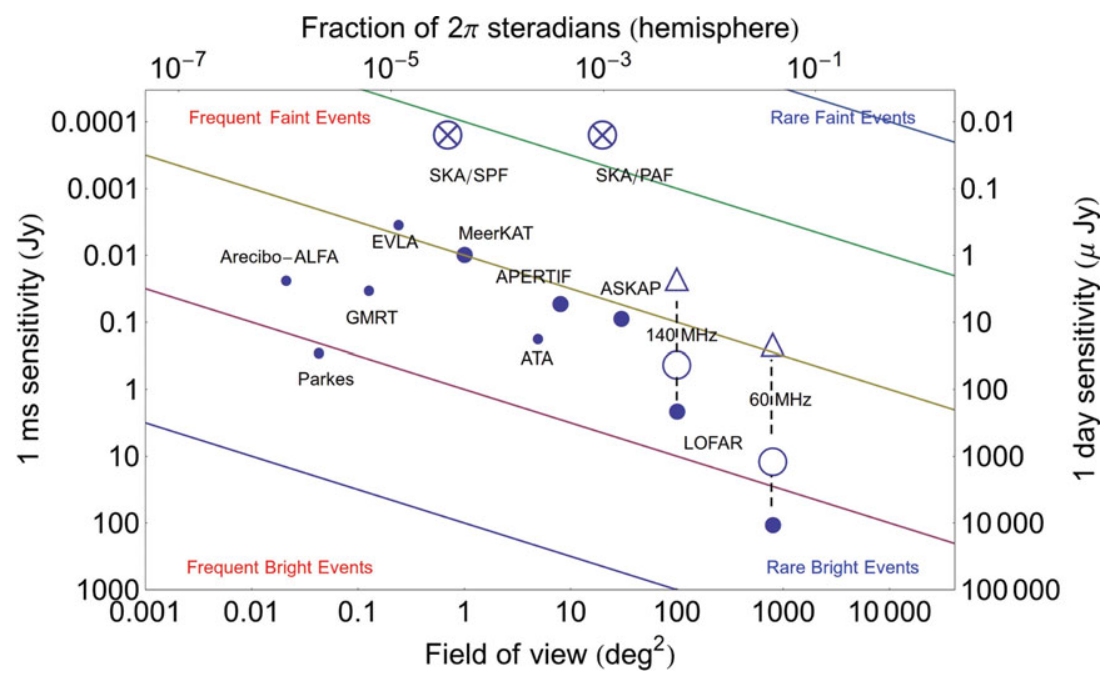

Figure 3. A comparison of sensitivity vs. field of view for a range of existing and planned radio telescopes. The solid lines represent a constant survey figure of merit (FoM, $\propto \Omega s^{-2}$ where $\Omega$ is the field of view and $s$ the sensitivity; smaller $s$ implies higher sensitivity). For LOFAR, the dots indicate the raw sensitivities, the open circles represent a spectral correction for a spectral index of -0.7 (where spectral index $\alpha$ is in the sense that $S_{\nu} \propto \nu^{\alpha}$ ), as is appropriate for optically thin synchrotron emission. The open triangles correspond to a correction for a spectral index of -2.0 , corresponding to the steepest (most aged) synchrotron sources, as well as some coherent radio sources such as pulsars and other flavours of neutron star. 


\section{Proposed Approach}

The LOFAR TKSP have considered several approaches for the detection of transients and variables, and propose a programme of targeted searches, wide-field blind surveys, and commensal searches for transients.

Targeted surveys. Within the TKSP there is a number of targeted observations and surveys planned (and in some cases, under way). They include observations of well-known high-energy astrophysics sources with variable radio counterparts (e.g. the binary SS 433, the pulsar PSR 0329+54, the blazar PKS 1510-089). They also include targets beyond the usual suspects, which may provide breakthroughs in some research areas (e.g. a search of nearby stars for radio bursts from 'hot Jupiters'). It is also our intention to probe transient parameter space by searching for concentrations of mass and/or exotic objects at a range of distances (and hence luminosities). They include globular clusters within our own galaxy, M31, the core of the Virgo cluster and beyond.

Wide-field blind surveys. An example of a wide-field blind survey planned for LOFAR is the zenith monitoring programme, which is a key component of the 'Radio Sky Monitor' (RSM) programme. Fig. 4 illustrates how a small number of 7-pointing tiles could cover the entire zenithal strip (Dec +54 for LOFAR), with maximum sensitivity (it peaks at the zenith for the dipoles). For example, with the LBAs at around $60 \mathrm{MHz}$, a 10 degree wide strip could be covered in 16 tiles, surveying around 2000 square degrees (10\% of the northern hemisphere; note that this is for fairly dense tiling). Thus a dedicated phase of RSM observing (which may be likely to happen once or twice per year for a few weeks, in coordination with other multiwavelength facilities) with $100 \%$ of resources spent on it, would correspond to $\sim 1.5 \mathrm{hr}$ on each field, resulting in an expected r.m.s. of a few mJy. At the lowest frequencies it only takes two more tiles to cover the entire northern galactic plane, which may well sample a different population of transients. At present we have been monitoring the single part of the zenithal field that contains the bright pulsar PSR $0329+54$ and using that to constrain and estimate the rate of low frequency transients (Bell, 2011). In fact AARTFAAC (Amsterdam-ASTRON Radio Transients Facility and Analysis Centre; PI Wijers) plans to use the LOFAR core in

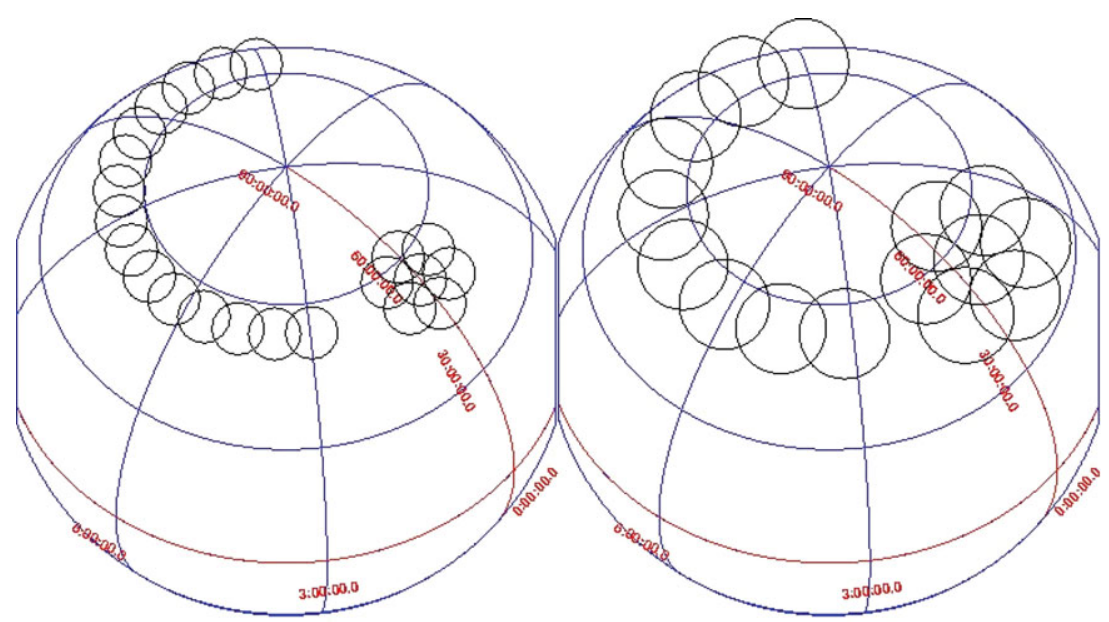

Figure 4. The proposed zenith monitoring programme for LOFAR. During intense periods of monitoring, some 2000 square degrees of the sky, centred on Dec +54 , could be imaged to $\sim \mathrm{mJy}$ sensitivity every $24 \mathrm{hr}$. This Figure demonstrates the enormous field of view of LOFAR. 
parallel with other observing programmes to perform a quasi-continuous, low-angularresolution survey of the entire northern hemisphere for transients.

Commensal surveys. Since the initial design of LOFAR it has been a stated goal of the TKSP that LOFAR data be imaged on short $(\leqslant 10 \mathrm{sec})$ time-scales in almost real time, in order to search for bright transients and variables. That functionality has yet to be implemented, but is in the design and commissioning plan for 2012. In the meantime the TKSP aims to search all other observations performed by LOFAR in a hunt for transients. That will naturally give us a range of wide-field and deep surveys (such as those planned by the Surveys and Epoch of Reionisation KSPs) with which to probe transient phase space. It is not unreasonable to assume that the entire northern hemisphere will effectively be surveyed several times per year, besides making deeper pointings with much higher cadence.

\section{Predicted Rates}

Until recently, the best estimates for predicted rates were around 0.1 sources $\mathrm{deg}^{-1}$ for two-epoch transients and a flux-density limit of $\sim$ mJy (Bell 2011, based on Bower et al. 2007); however, according to Frail et al. (2011) that may be an overestimate by up to an order of magnitude. For the zenith monitoring programme outlined above, we could have $2000 \mathrm{deg}^{2}$ surveyed to approximately the same r.m.s. per $24 \mathrm{hr}$, resulting in a transient detection rate from this programme alone of 20 (Frail) to 200 (Bower) events per day. Considering commensal searches of all data, which may on any given day be going wide, or deep, or some combination, we can conservatively expect more than ten high-significance transient events per day. One major unknown is the fraction of events that will prove to be repeaters, so the true number of distinct astrophysical variables found may be lower. It is our stated policy to report these events as widely as possible, probably via some version of VOEvent (Seaman et al. 2011), for example skyalert.org.

\section{Summary}

LOFAR has a wide range of diverse capabilities - multi-beaming, simultaneous timing and imaging modes, splitting the array between large numbers of individual stations, reimaging past observations with the TBBs - all of which are ideally suited for exploring transient parameter space. Just as importantly, there is the will to support this science: a Key Science Project is dedicated precisely to that exploration. According to current estimates, it should find many thousands of interesting radio transients per year, thus furnishing a huge target list for multiwavelength follow-ups and providing new tests of our ability to detect, classify and report automatically such events efficiently.

\section{References}

Bell, M. E. 2011, PhD thesis, University of Southampton

Bower, G. C., et al. 2007, ApJ, 6, 346

Frail, D. A., et al. 2011, ApJ, submitted

Seaman, R., et al. 2011, IVOA Recommendation, arXiv:1110.0523

Stappers, B. W., Hessels, J. W. T., Alexov, A., et al. 2011, A\& A, 530, 80

LOFAR: www. lofar .org

LOFAR Transients Key Science Project: www.transientskp.org 